Original Paper http://ajol.info/index.php/ijbcs http://indexmedicus.afro.who.int

\title{
Elimination de polluants des déchets liquides d'une unité de production de sucre par des argiles naturelles de Côte d'Ivoire
}

\author{
Atolé Brice Bienvenu KEDI ${ }^{1 *}$, Seka Simplice KOUASSI ${ }^{1}$, Vamoussa COULIBALY ${ }^{2}$ et \\ Joseph SEI ${ }^{2}$ \\ ${ }^{1}$ Laboratoire des Sciences et Technologies de l'Environnement, UFR Environnement, Université Jean \\ Lorougnon GUEDE, BP 150 Daloa, Côte d'Ivoire. \\ ${ }^{2}$ Laboratoire de Constitution et de Réaction de la Matière, UFR SSMT, Université Félix Houphouët-Boigny, 22 \\ BP 582 Abidjan 22, Côte d'Ivoire. \\ *Auteur correspondant, E-mail : brice.kedi@ujlg.edu.ci; Tel. : (+225) 0747938992
}

\author{
Received: 13-11-2020 \\ Accepted: 09-04-2021 \\ Published: 30-04-2021
}

\section{RESUME}

L'impact des déchets liquides industriels sur l'environnement demeure une réalité et une menace pour la qualité des eaux souterraines et de surfaces. Parmi les méthodes de dépollution existantes, l'adsorption des polluants par les argiles naturelles reste une méthode moins couteuse, disponible et facilement utilisable. La plupart des études sur l'aptitude des argiles à éliminer les polluants est faite avec des déchets liquides artificiels dont les concentrations sont maitrisées. L'objectif de cette étude était d'éliminer les polluants dans les déchets liquides d'une unité industrielle agronomique (UIA) de production de sucre par deux argiles naturelles de Côte d'Ivoire. Trois sources de déchets liquides provenant des activités de laboratoire d'analyse des sols, de jus de canne et de canne à sucre de l'UIA. Le traitement aux argiles a montré des taux d'élimination à divers degrés des polluants. Les paramètres suivants ont été mesuré avant et après le traitement aux argiles : pH, conductivité, turbidité, phosphore total, azote total, cuivre, zinc, plomb et mercure. L'argile de Katiola présente des aptitudes plus élevées à éliminer les polluants, comparée à l'argile d'Anyama. Cette étude confirme l'intérêt de l'utilisation des argiles pour la dépollution.

(C) 2021 International Formulae Group. All rights reserved.

Mots clés : Eaux usées, métaux lourds, pollution, argiles, adsorption.

\section{Removal of liquid waste pollutants from a sugar production unit using natural clays from Côte d'Ivoire}

\begin{abstract}
The impact of industrial liquid waste on the environment remains a reality and a threat to groundwater and surface water quality. Among the existing depollution methods, the pollutants adsorption on natural clays remains a less expensive, available and easily usable method. Most studies on the ability of clays to remove pollutants are done with artificial liquid wastes whose concentrations are controlled. The objective of this study was to eliminate the pollutants in the liquid waste of an industrial agronomic sugar production unit using two natural clays from Côte d'Ivoire. Three sources of liquid wastes from the UIA's analysis laboratory activities. Treatment with clays showed varying degrees of removal of pollutants. The following parameters were measured
\end{abstract}


before and after the clay treatment: $\mathrm{pH}$, conductivity, turbidity, total suspended solids, total phosphorus, total nitrogen, copper, zinc, lead and mercury. The values of these parameters are compared to the national standard. Katiola clay exhibits higher abilities to remove pollutants compared to Anyama clay. This study confirms the value of using clays for depollution.

(C) 2021 International Formulae Group. All rights reserved.

Keywords: Wastewater, heavy metals, pollution, clays, adsorption.

\section{INTRODUCTION}

La pollution des effluents industrielles et leur impact sur notre milieu de vie demeurent une réalité et une menace à moyen et à long terme pour la qualité des eaux de surfaces et des sols (Gnagne et al., 2015; Ouedraogo et al., 2019). Le problème de la pollution industrielle s'accentue dans les pays en voie de développement avec l'installation croissante des usines et le manque de système adéquat pour le traitement des déchets liquides engendrés (Dongo et al., 2013).

La prise de conscience de l'impact environnemental des effluents industriels a fait de la recherche sur le traitement des déchets liquides une priorité. Plusieurs techniques de dépollution ont été développées mais engendrent eux même des produits toxiques et ont des coûts élevés. Ces techniques sont l'extraction de solvant, l'évaporation, la précipitation chimique, l'échange d'ions, le traitement électrochimique, la technologie de filtration membranaire, les technologies émergentes de systèmes organo-métallique et les procédés d'oxydation avancés (Gu et al., 2019). La technique d'adsorption des polluants par les adsorbants moins couteux, disponibles et facilement utilisables comme les argiles, constitue un grand intérêt (Bailey et al., 1999; Mockovčiaková et al., 2010) pour la lutte antipollution. Plusieurs études ont rapporté l'efficacité des argiles à adsorber les polluants des déchets liquides (Hu and Luo, 2010; Ren et al., 2014; Sorgho et al., 2019). Très souvent dans ces études, l'évaluation de la capacité des argiles à éliminer les polluants se fait avec des minéraux argileux et des eaux usées artificielles dont la concentration des polluants est contrôlée. Il existe très peu d'étude avec des déchets liquides industrielles réels contenant plusieurs polluants avec des concentrations élevées.

La Côte d'Ivoire dispose de nombreux sites de matériaux argileux dont l'usage peut être envisagée dans la protection de l'environnement. Des investigations ont montré que les argiles de Katiola et d'Anyama pourraient présenter des caractéristiques intéressantes pour leur usage dans la dépollution (Kpangni et al., 2008; Coulibaly et al., 2013, 2018).

L'objectif de ce travail est d'éliminer les polluants des déchets liquides produits par une unité industrielle de production de sucre de Côte d'Ivoire par des argiles naturelles locales. Différents paramètres organiques et inorganiques sont mesurés avant et après traitement de ces déchets liquides par deux argiles naturelles et sont comparés aux valeurs de la norme ivoirienne de rejets de déchets liquides (MEF, 2008).

\section{MATERIEL ET METHODES \\ Zone d'étude}

Les déchets liquides de notre étude sont issus des laboratoires de l'Unité Agricole Intégrée (UAI) de Zuénoula. Cette unité est située à $25 \mathrm{~km}$ de Zuénoula au Centre-Ouest de la Côte d'Ivoire. Elle est géographiquement localisée entre $7^{\circ} 30^{\prime}$ et $7^{\circ} 40$ de latitude Nord, et entre $6^{\circ} 5$ et $6^{\circ} 15$ de longitude Ouest, et située à $209 \mathrm{~m}$ d'altitude par rapport à la mer.

\section{Déchets liquides des laboratoires}

Les déchets liquides sont produits par deux laboratoires (agronomique et usine) de l'UAI lors de la campagne de production qui dure environ sept mois dans l'année. Les rejets 
liquides, après les analyses du laboratoire agronomique sur la canne à sucre, les feuilles et les sols, constituent les échantillons de déchets liquides nommés A. Les déchets liquides issus des analyses du laboratoire de l'usine sur les jus de canne produits sont les échantillons nommés B. Les eaux de rinçage de la verrerie du laboratoire de l'usine, après les analyses, constituent les échantillons $\mathrm{C}$.

\section{Méthode d'échantillonnage}

La collecte des échantillons s'est faite une fois par semaine sur quatre semaines. Des échantillons composites ont été formés à partir des échantillons de chacune des quatre semaines pour chacune des sources de déchets liquides $\mathrm{A}, \mathrm{B}$ et $\mathrm{C}$. Ces échantillons ont été conservés au congélateur entre $2{ }^{\circ} \mathrm{C}$ et $5{ }^{\circ} \mathrm{C}$, dans des bouteilles en polyéthylène préalablement nettoyés et acidifiés à $\mathrm{pH} 2$ avec de l'acide chlorhydrique $(\mathrm{HCl})$ concentré. Afin de minimiser toute contamination éventuelle, l'échantillonnage s'est fait avec un grand soin.

\section{Traitement des déchets liquides avec les argiles}

Une partie des échantillons composites A, B et C a été utilisée pour le traitement avec chacune des deux argiles. $10 \mathrm{~g}$ d'argile ont été pesés et ajoutées à $500 \mathrm{~mL}$ d'échantillon de déchets liquides de sorte à avoir $20 \mathrm{~g}$ pour un litre de déchet liquide. Les mélanges ont été agités pendant une heure à l'aide d'agitateur magnétique de type HI-200M. Les solutions d'argiles ont été centrifugées à $4000 \mathrm{rpm}$ à l'aide d'une centrifugeuse Sigma-2-16P. Le surnageant a été recueilli dans des bouteilles en polyéthylène préalablement nettoyées et acidifiées à $\mathrm{pH} 2$, avec de l'acide chlorhydrique ( $\mathrm{HCl})$ concentré, et conservés au congélateur entre $2{ }^{\circ} \mathrm{C}$ et $5{ }^{\circ} \mathrm{C}$ pour les analyses.

\section{Analyse au laboratoire}

La température, la conductivité, la matière en suspension (MES) et la turbidité ont été mesurés au laboratoire de l'usine après l'échantillonnage et avant la conservation des échantillons au congélateur. La température et le $\mathrm{pH}$ ont été mesurés à l'aide d'un appareil de type knick PH-Meter 765 Calamatic. La conductivité a été déterminée à l'aide du multimètre Consort C933. La turbidité et la matière en suspension (MES) ont été évaluées avec le multimètre Nalco DR/ 890 Colorimeter.

Les concentrations des paramètres inorganiques et organiques que sont l'azote total, le phosphore total, le mercure $(\mathrm{Hg})$, le plomb $(\mathrm{Pb})$, le zinc $(\mathrm{Zn})$ et le cuivre $(\mathrm{Cu})$ ont été mesurées au Laboratoire National d'Essais de qualité de Métrologies et d'Analyses (LANEMA) d'Abidjan. Le dosage des différents métaux a été effectué au moyen d'un Spectromètre d'Absorption Atomique (SAA) BK-AA320N. Les méthodes d'analyses utilisées ou le matériel sont consignées dans le Tableau 1. Le taux de réduction des paramètres est calculé en faisant le rapport des valeurs, après et avant traitement aux argiles multiplié par cent.

\section{Echantillons d'argiles}

Les deux argiles utilisées dans cette étude sont des argiles naturelles prélevées dans deux villes de Côte d'Ivoire (Anyama et Katiola). Elles ont été fournies par le laboratoire de Constitution et de Réaction de la Matière de l'Université Félix HouphouëtBoigny d'Abidjan (Côte d'Ivoire). Ces échantillons ont été broyés dans un mortier en porcelaine et tamisés à $100 \mu \mathrm{m}$. Les principaux minéraux argileux et la surface spécifique de ces argiles sont dans le Tableau 2.

Les minéraux argileux dominants sont la kaolinite, l'illite et les smectites dans l'argile d'Anyama et l'illite, la chlorite et les smectites dans l'argile de Katiola. Les pourcentages de minéraux argileux sont de 50,13\% et $64,02 \%$ respectivement pour les argiles d'Anyama et de Katiola. 
Tableau 1: Méthodes d'analyses utilisées et appareillage.

\begin{tabular}{lll}
\hline Paramètres & Unité & Materiel ou methodes utilisés \\
\hline $\mathrm{pH}$ & - & Knick-765 Laboratory pH Meter \\
Conductivité & $(\mu \mathrm{S} / \mathrm{Cm})$ & multimètre Consort C933 \\
MES & $(\mathrm{mg} / \mathrm{L})$ & multimètre Nalco DR/ 890 Colorimeter \\
Turbidité & $(\mathrm{NTU})$ & multimètre Nalco DR/ 890 Colorimeter \\
Phosphore total & $(\mathrm{mg} / \mathrm{L})$ & ISO 6878 v 2004 \\
Azote total & $(\mathrm{mg} / \mathrm{L})$ & ISO 5663 v 1984 \\
Cuivre & $(\mathrm{mg} / \mathrm{L})$ & Spectromètre d'Absorption Atomique BK-AA320N/Rodier 9 \\
Zince edition \\
Plomb & $(\mathrm{mg} / \mathrm{L})$ & Spectromètre d'Absorption Atomique BK-AA320N/ISO 15586 v 2003 \\
Mercure & $(\mathrm{mg} / \mathrm{L})$ & Spectromètre d'Absorption Atomique BK-AA320N/ISO 15586 v 2003 \\
\hline
\end{tabular}

Tableau 2: Constituants minéraux des argiles utilisées.

\begin{tabular}{lcc}
\hline & Argile d'Anyama & Argile de Katiola \\
\hline Illite (\%) & 14,94 & 26,31 \\
Chlorite (\%) & - & 23,31 \\
Smectites (\%) & 12,81 & 14,40 \\
Kaolinite (\%) & 22,38 & - \\
Rutile (\%) & - & 1,03 \\
Quartz (\%) & 17,11 & 29,73 \\
Total minéraux argileux (\%) & 50,13 & 64,02 \\
Surface spécifique $\left(\mathrm{m}^{2} / \mathrm{g}\right)$ & 29 & 36 \\
\hline
\end{tabular}




\section{RESULTATS}

\section{Potentiel d'hydrogène $(\mathrm{pH})$}

Les résultats des mesures de $\mathrm{pH}$, de la conductivité, de la turbidité et de MES, avant et après traitement aux argiles sont représentés sur la Figure 1. Les valeurs de $\mathrm{pH}$ des trois sources de déchets liquides avant traitement aux argiles sont comprises entre 4,37 et 4,54. Après le traitement aux argiles, les valeurs de $\mathrm{pH}$ n'ont pas beaucoup évolué car elles sont comprises entre 4,2 et 4,7 . Les valeurs de $\mathrm{pH}$ montrent que ces déchets liquides, avant et après le traitement aux argiles, sont acides et ne sont pas conformes aux normes ivoiriennes, qui suggèrent que les valeurs de $\mathrm{pH}$ des rejets soient comprises entre 5,5 et 8,5 .

\section{Conductivité}

Les déchets liquides brutes $\mathrm{A}_{0}$ présentent une forte conductivité (11820 $\mu \mathrm{S} / \mathrm{Cm})$ qui fait 3,5 fois celle de $\mathrm{B}_{0}(3300$ $\mu \mathrm{S} / \mathrm{Cm}$ ), bien que cette dernière soit élevée. Par contre, les déchets liquides brutes $\mathrm{C}_{0}$ ont une faible conductivité $(540 \mu \mathrm{S} / \mathrm{Cm})$, comparée aux deux autres. Avec les argiles, la conductivité de $\mathrm{A}_{0}$ est passée de 11820 à 2313 et $480 \mu \mathrm{S} / \mathrm{Cm}$ respectivement avec les argiles d'Anyama et de Katiola, soit une réduction de $80 \%$ et $96 \%$ respectivement. Quant à la conductivité de l'échantillon $\mathrm{B}_{0}$, elle a été légèrement réduite, passant de $3300 \mu \mathrm{S} / \mathrm{Cm}$ à $2760 \mu \mathrm{S} / \mathrm{Cm} \quad$ avec l'argile d'Anyama et à $2838 \mu \mathrm{S} / \mathrm{Cm}$ avec l'argile de Katiola, soit une réduction de $16 \%$ et $14 \%$ respectivement. En revanche, la conductivité de l'échantillon $\mathrm{C}_{0}$, qui était la moins élevée des trois, a plutôt augmenté après le traitement aux argiles. Ainsi, la conductivité est passée de $540 \mu \mathrm{S} / \mathrm{Cm}$ à 804 $\mu \mathrm{S} / \mathrm{Cm}$ avec l'argile d'Anyama et à $573 \mu \mathrm{S} / \mathrm{Cm}$ avec l'argile de Katiola.

\section{Turbidité}

Les déchets $\mathrm{A}_{0}$ (5745 NTU) sont les plus troubles, suivi des déchets $\mathrm{B}_{0}(2160 \mathrm{NTU})$ avec une turbidité deux fois moins élevée. Les déchets $\mathrm{C}_{0}$ sont les moins troubles avec une turbidité de 262 NTU. Le traitement aux argiles a réduit fortement les valeurs de turbidité des trois déchets liquides. La turbidité de $\mathrm{A}_{0}$ a été réduite de $98 \%$ et $99 \%$ respectivement avec les argiles d'Anyama et de Katiola. Quant aux déchets liquides $\mathrm{B}_{0}$, la réduction était de $92 \%$ et de $96 \%$ avec l'argile d'Anyama et de Katiola. La turbidité du moins trouble des déchets liquides $\mathrm{C}_{0}$, a été réduite de $86 \%$ et de $63 \%$ avec les argiles d'Anyama et Katiola respectivement. La réduction de la turbidité des déchets liquides est visible sur la Figure 2 avec des couleurs plus foncées pour les échantillons brutes non traités et des couleurs plus claires pour les échantillons traités aux argiles.

\section{Matière en suspension (MES)}

Les valeurs de MES des trois déchets liquides sont élevées et supérieures à la norme ivoirienne $(150 \mathrm{mg} / \mathrm{L})$. Les valeurs des déchets $\mathrm{A}_{0}(4035 \mathrm{mg} / \mathrm{L})$ et $\mathrm{B}_{0}(2150 \mathrm{mg} / \mathrm{L})$ sont bien plus élevées que la norme nationale avec des facteurs de 27 et 14 respectivement. Les déchets $\mathrm{C}_{0}$ ont une valeur de $180 \mathrm{mg} / \mathrm{L}$. Le traitement aux argiles a considérablement réduit la matière en suspension (MES) dans les trois échantillons de déchets liquides. Les nouvelles valeurs de MES, après traitement, sont inférieures à la limite maximale (150 $\mathrm{mg} / \mathrm{L}$ ) de la norme ivoirienne, donc conforme à cette norme. Les pourcentages de réduction de MES dans les déchets $\mathrm{A}_{0}$ est d'environ $98 \%$ avec les deux argiles. Avec les déchets $\mathrm{B}_{0}$, la réduction est de 95\% avec l'argile d'Anyama et $97 \%$ avec l'argile de Katiola. Quant aux déchets $\mathrm{C}_{0}$, le taux de réduction est de $87 \%$ et $62 \%$ respectivement avec les argiles d'Anyama et de Katiola.

\section{Phosphore total et Azote total}

Le taux de phosphore total des déchets liquides $\mathrm{A}_{0}(35,64 \mathrm{mg} / \mathrm{L})$ et $\mathrm{B}_{0}(24,74 \mathrm{mg} / \mathrm{L})$ dépasse largement celui recommandé par la norme nationale $(15 \mathrm{mg} / \mathrm{L})$ sauf dans le cas des déchets $\mathrm{C}_{0}$ où la concentration est de $4,7 \mathrm{mg} / \mathrm{L}$. Le constat est le même pour la mesure de 
l'Azote total. Seul l'échantillon $\mathrm{C}_{0}$ respecte la norme ivoirienne $(50 \mathrm{mg} / \mathrm{L})$ avec une valeur de 2,10 mg/L. Les deux autres échantillons $A_{0}$ $(166,88 \mathrm{mg} / \mathrm{L})$ et $\mathrm{B}_{0}(183,68 \mathrm{mg} / \mathrm{L})$ ont des valeurs qui dépassent largement (plus de trois fois) celle autorisée.

Le traitement aux argiles a fait baisser à divers degrés la concentration de phosphore total et d'azote total dans les déchets liquides. Ce traitement a réduit (entre 85 et $97 \%$ de réduction) la concentration de phosphore total et d'azote total dans les déchets $\mathrm{B}_{1}$ et $\mathrm{B}_{2}$, et leur a permis d'être conforme à la norme. Par contre dans le cas des déchets $A_{1}$ et $A_{2}$, les concentrations de phosphore total et d'azote total ont été réduites (entre 16 et $43 \%$ ) mais les valeurs restent toujours supérieures aux valeurs recommandées par la norme ivoirienne. Bien qu'étant déjà conforme à la norme, les déchets C ont connu une forte baisse (entre 83 et $93 \%$ de réduction) de sa quantité de phosphore total et d'azote total.

\section{Eléments métalliques}

L'analyse des trois déchets liquides a révélé la présence de métaux lourds comme le cuivre, le zinc, le plomb, le mercure. Les concentrations en cuivre et en zinc des trois déchets liquides varient respectivement entre 0,15 et $0,17 \mathrm{mg} / \mathrm{L}$, et entre 0,42 et $0,44 \mathrm{mg} / \mathrm{L}$. Ces valeurs sont nettement inférieures aux limites maximales recommandées par la norme nationale qui sont de $0,5 \mathrm{mg} / \mathrm{L}$ pour le cuivre, et de $2 \mathrm{mg} / \mathrm{L}$ pour le zinc. En revanche, les concentrations en plomb et en mercure des trois déchets liquides dépassent largement les valeurs limites maximales, qui sont de 0,5 $\mathrm{mg} / \mathrm{L}$ pour le plomb et $0,2 \mathrm{mg} / \mathrm{L}$ pour le mercure.

Le traitement aux argiles a eu des effets divers sur la concentration en cuivre et en zinc, avec une réduction plus élevée des concentrations par l'argile de Katiola par rapport à l'argile d'Anyama. Le taux de réduction des concentrations en cuivre de l'argile de Katiola est de $63 \%$ contre $14 \%$ pour les déchets A, $27 \%$ contre $22 \%$ pour les déchets B et 54 contre $35 \%$ pour les déchets C. De même, la réduction de la concentration en zinc de l'argile de Katiola comparée à celle d'Anyama, est de $38 \%$ contre $61 \%$ pour les déchets A, $20 \%$ contre $8 \%$ pour les déchets B et $2 \%$ contre $1,5 \%$ pour les déchets $\mathrm{C}$.

Quant aux concentrations en plomb et en mercure, elles ont été réduites à divers degrés par les deux argiles. Dans les déchets A, la concentration de plomb a été fortement réduite de $81,45 \mathrm{mg} / \mathrm{L}$ à $5,39 \mathrm{mg} / \mathrm{L}$ avec l'argile d'Anyama et à $3,88 \mathrm{mg} / \mathrm{L}$ avec l'argile de Katiola, soient des réductions de $93 \%$ et 95\% respectivement. Dans les déchets B et C la concentration de plomb a été réduite avec des taux variant entre $29 \%$ et $37 \%$ avec les deux argiles sauf dans le cas des déchets $\mathrm{C}$ avec l'argile de Katiola où le taux de réduction est de $77 \%$. Bien que les concentrations en plomb aient été réduites dans les trois déchets, elles restent toujours supérieures à la valeur maximale $(0,5 \mathrm{mg} / \mathrm{L})$ recommandée par la norme.

La concentration en mercure dans les trois échantillons de déchets liquides a été faiblement réduite avec des taux variant entre $5 \%$ et $19 \%$ avec les deux argiles. Dans l'échantillon A, la concentration de mercure est passée de $8,33 \mathrm{mg} / \mathrm{L}$ à $7,75 \mathrm{mg} / \mathrm{L}$ avec l'argile d'Anyama et 7,2 mg/L avec l'argile de Katiola, soient des réductions de $7 \%$ et $14 \%$ respectivement. Dans l'échantillon B, cette concentration est passée de $6,8 \mathrm{mg} / \mathrm{L}$ à 6,3 $\mathrm{mg} / \mathrm{L}$ et $5,86 \mathrm{mg} / \mathrm{L}$, soient des taux de réduction de $7 \%$ et $14 \%$ avec l'argile d'Anyama et de Katiola respectivement. Quant à la concentration de mercure dans l'échantillon $\mathrm{C}$, elle est passée de $5,2 \mathrm{mg} / \mathrm{L}$ à $4,95 \mathrm{mg} / \mathrm{L}$ et $4,22 \mathrm{mg} / \mathrm{L}$, soient des taux de réduction de $5 \%$ et $19 \%$ respectivement avec l'argile d'Anyama et l'argile de Katiola. Les concentrations en mercure avant et après traitement des trois échantillons restent supérieures à la valeur maximale $(0,2 \mathrm{mg} / \mathrm{L})$ recommandée par la norme ivoirienne. 

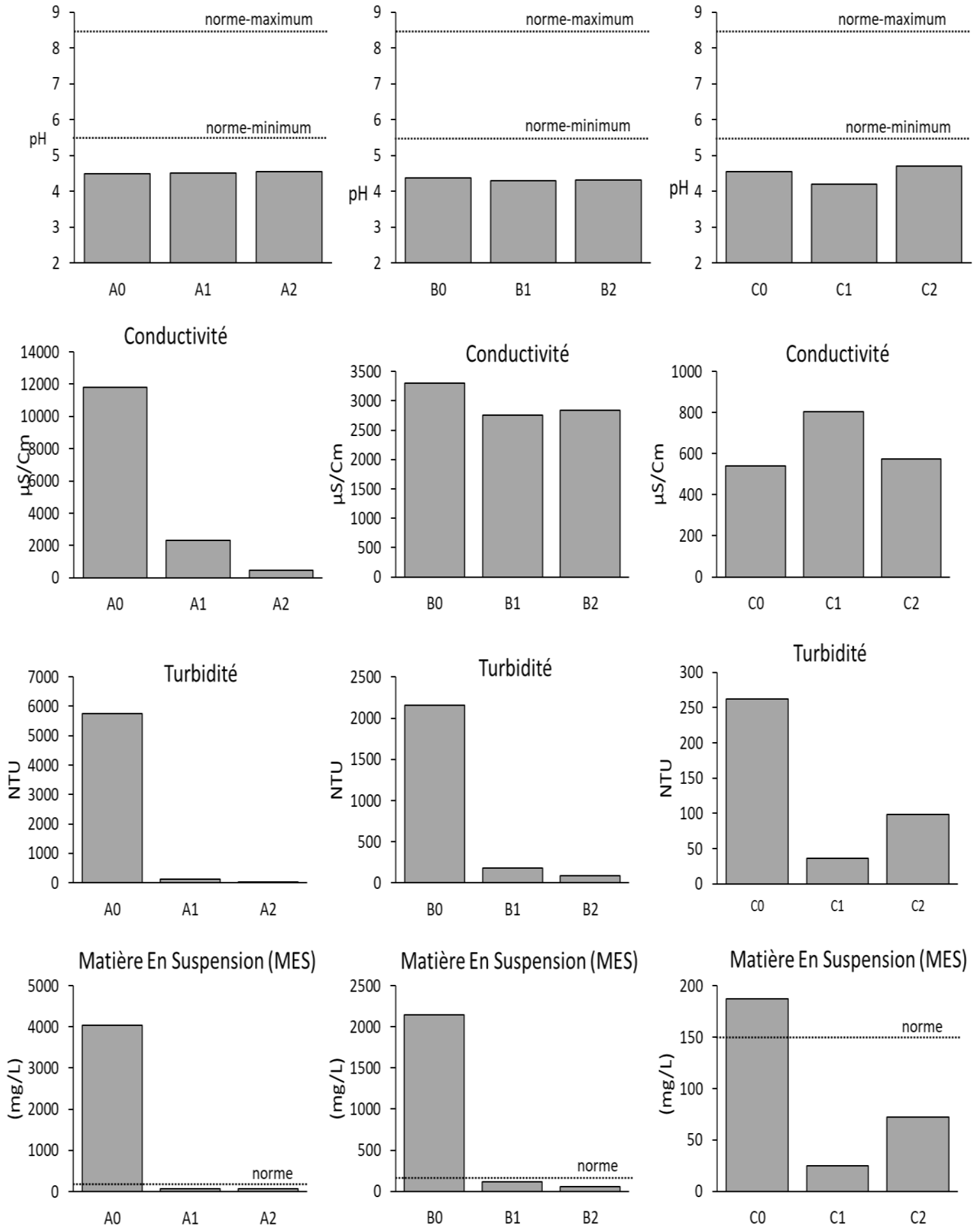

Figure 1: $\mathrm{pH}$, Conductivité, Turbidité Matière En Suspension (MES) des déchets liquides avant $\left(\mathrm{A}_{0}, \mathrm{~B}_{0}\right.$ et $\left.C_{0}\right)$ et après traitement avec les argiles d'Anyama $\left(A_{1}, B_{1}\right.$ et $\left.C_{1}\right)$, de Katiola $\left(A_{2}, B_{2}\right.$ et $\left.C_{2}\right)$ et la norme Ivoirienne. 

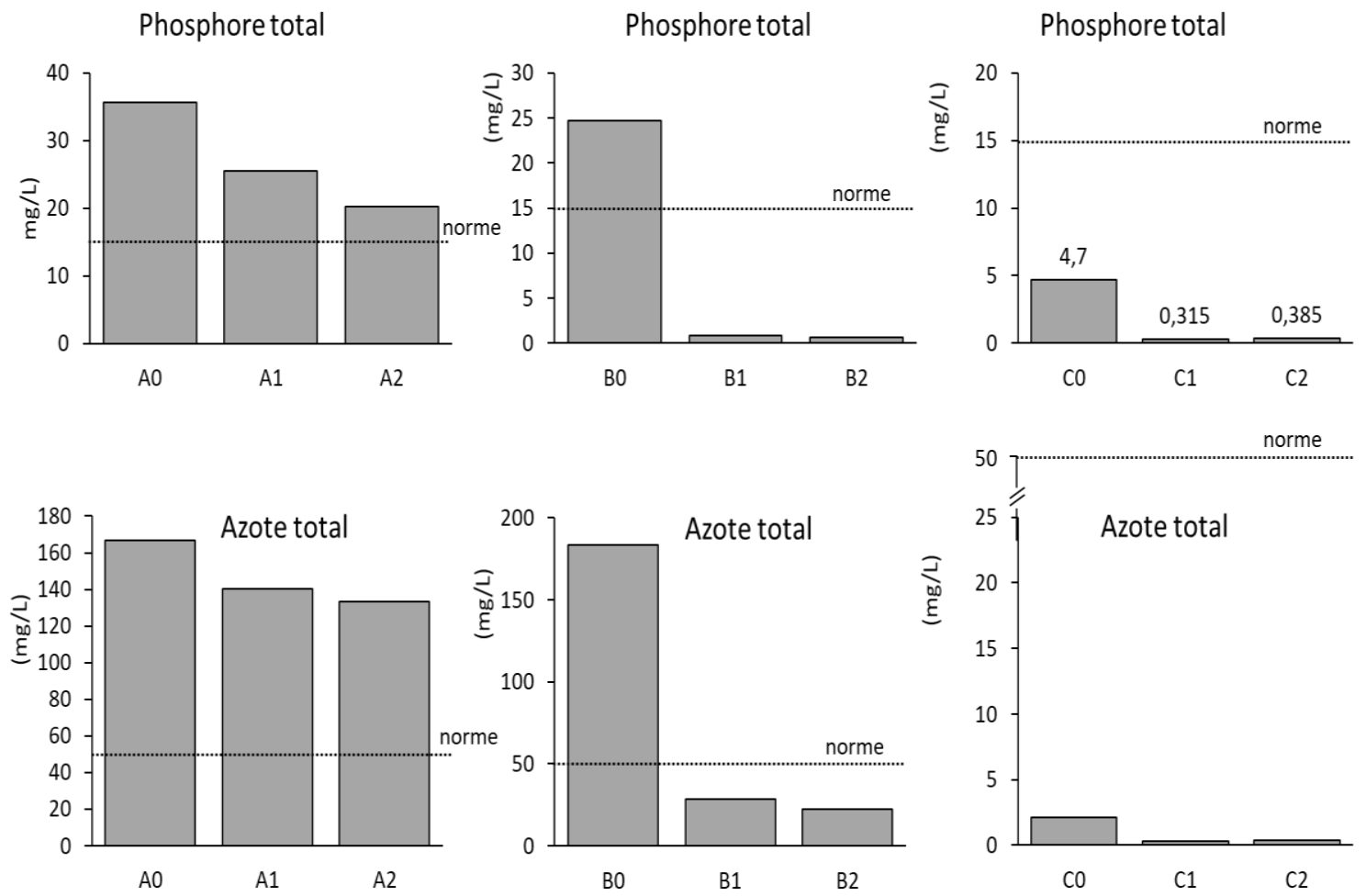

Figure 2: Concentration en phosphore total et en azote total des déchets liquides avant $\left(A_{0}, B_{0}\right.$ et $\left.C_{0}\right)$ et après traitement avec les argiles d'Anyama $\left(A_{1}, B_{1}\right.$ et $\left.C_{1}\right)$, de Katiola $\left(A_{2}, B_{2}\right.$ et $\left.C_{2}\right)$ et la norme Ivoirienne.

Tableau 3: Pourcentage de réduction des paramètres physico-chimiques avec les argiles d'Anyama (A1, B1 et C1 ) et de Katiola (A2, B2 et C2).

\begin{tabular}{lcccccc}
\hline & $\mathbf{A}_{\mathbf{1}}$ & $\mathbf{A}_{\mathbf{2}}$ & $\mathbf{B}_{\mathbf{1}}$ & $\mathbf{B}_{\mathbf{2}}$ & $\mathbf{C}_{\mathbf{1}}$ & $\mathbf{C}_{\mathbf{2}}$ \\
\hline $\mathrm{pH}$ & $-0,67$ & $-1,34$ & 1,83 & 1,14 & 7,49 & $-3,52$ \\
conductivité & 80,43 & 95,94 & 16,36 & 14,00 & $-48,89$ & $-6,11$ \\
Turbidité & 97,91 & 99,48 & 91,62 & 96,11 & 86,26 & 62,60 \\
MES & 98,14 & 98,41 & 94,65 & 97,44 & 86,63 & 61,50 \\
Phosphore total & 28,34 & 43,07 & 96,74 & 97,45 & 93,30 & 91,81 \\
Azote total & 15,82 & 20,13 & 84,53 & 87,75 & 84,67 & 82,86 \\
Cuivre & 14,29 & 62,59 & 21,91 & 27,28 & 34,69 & 54,26 \\
Zinc & 61,48 & 37,97 & 7,87 & 20,49 & 1,51 & 2,08 \\
Plomb & 93,38 & 95,24 & 28,95 & 37,36 & 36,73 & 77,06 \\
Mercure & 6,96 & 13,57 & 7,35 & 13,82 & 4,81 & 18,85 \\
\hline
\end{tabular}



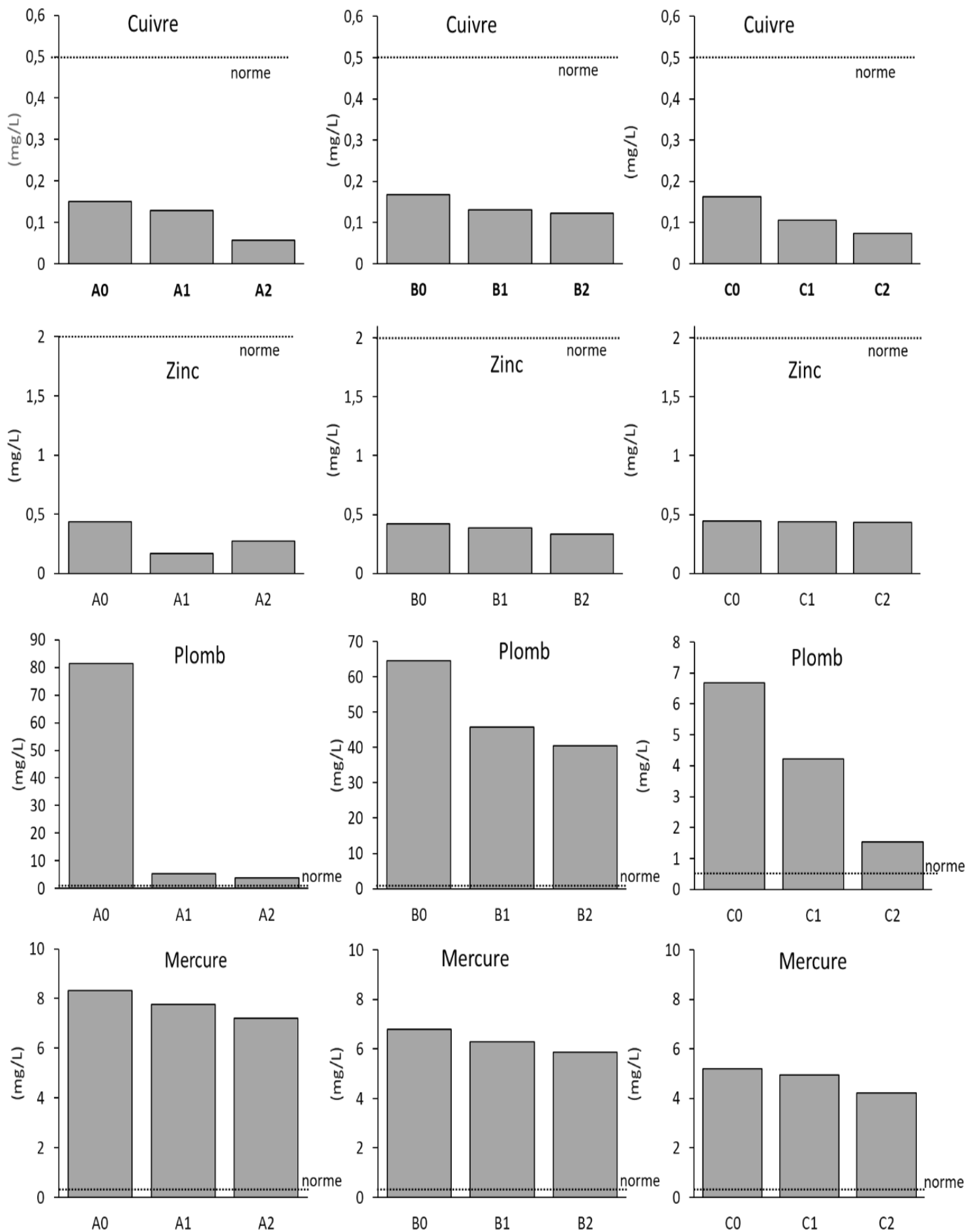

Figure 3: Concentration en métaux lourds des déchets liquides avant $\left(\mathrm{A}_{0}, \mathrm{~B}_{0}\right.$ et $\left.\mathrm{C}_{0}\right)$ et après traitement avec les argiles d'Anyama $\left(A_{1}, B_{1}\right.$ et $\left.C_{1}\right)$, de Katiola $\left(A_{2}, B_{2}\right.$ et $\left.C_{2}\right)$ et la norme Ivoirienne. 

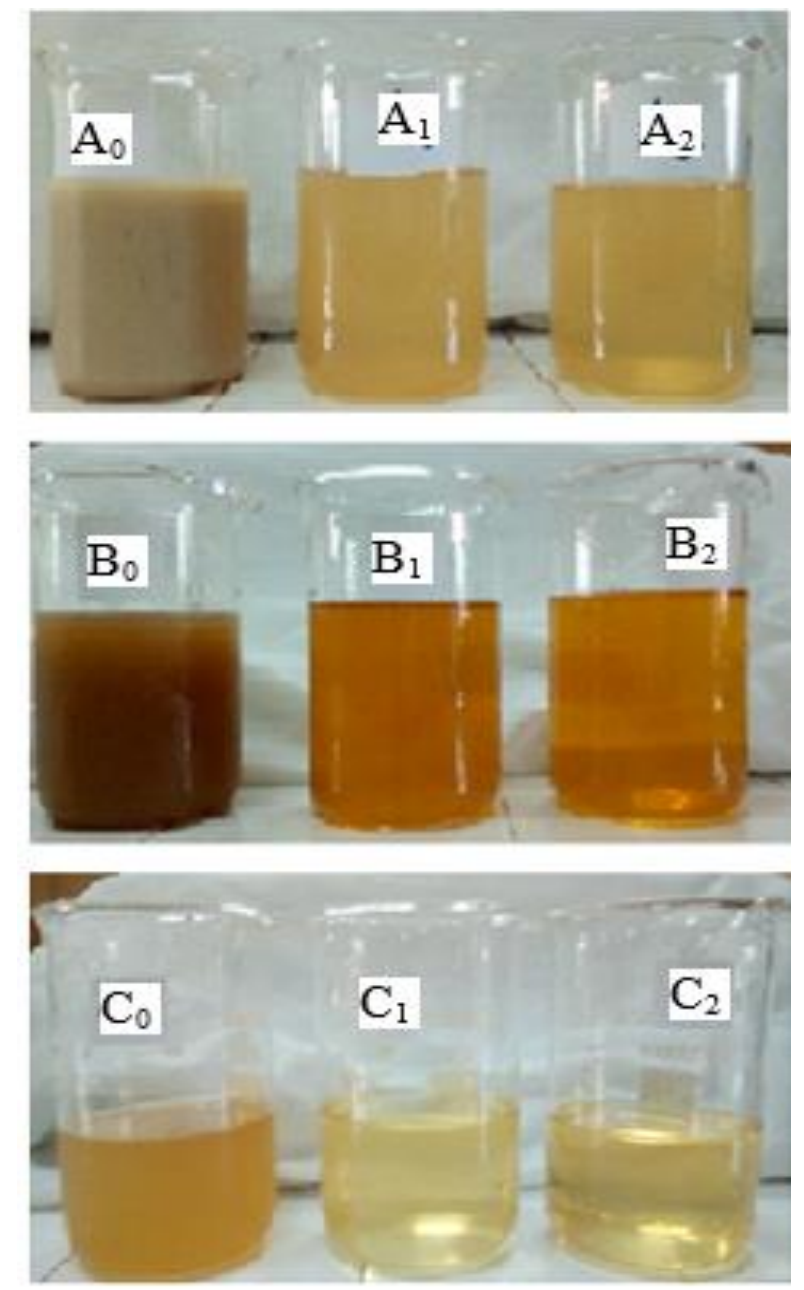

Figure 4: Echantillons de déchets liquides avant $\left(\mathrm{A}_{0}, \mathrm{~B}_{0}\right.$ et $\left.\mathrm{C}_{0}\right)$ et après traitement avec les argiles d'Anyama $\left(A_{1}, B_{1}\right.$ and $\left.C_{1}\right)$ et de Katiola $\left(A_{2}, B_{2}\right.$ et $\left.C_{2}\right)$.

\section{DISCUSSION}

Les argiles sont connues pour leur efficacité à éliminer les métaux toxiques et les autres polluants dans les eaux usées (Bhattacharyya and Gupta, 2008). Cette efficacité est due à leur capacité d'échange cationique, à leur grande surface spécifique et à leur charge négative pour la plupart des argiles (Yuan et al., 2013) qui leur confèrent de forte capacité d'adsorption. Les résultats ont montré une réduction des polluants organiques et inorganiques des déchets liquides à divers degrés. L'efficacité dans l'élimination des polluants de cette étude dépendait en partie des argiles. De façon générale l'argile de Katiola avait des taux d'élimination de polluants plus élevées que ceux de l'argile d'Anyama. En effet, cette argile est riche en minéraux de type $2: 1$ tels que les smectites $(14,40 \%)$ et les chlorites $(23,31 \%)$ contrairement à l'argile d'Anyama où les minéraux dominants sont de type $1: 1$ (kaolinite $(22,38 \%)$, illites $(14,94 \%)$ ). La composition des deux argiles explique les différences d'élimination des polluants, car les argiles de type 2 :1 ont des capacités d'adsorption plus élevées que celles de type $1: 1$ (Gu et al., 2019). 
Tous les déchets liquides ont un $\mathrm{pH}$ acide et le traitement aux deux argiles n'a pas influencé leur $\mathrm{pH}$. La quantité des ions $\mathrm{H}^{+}$ responsables de l'acidité des déchets liquide n'a pas été influencée par la présence des deux argiles. La conductivité a été fortement réduite dans le déchets liquides $\mathrm{A}$ et moins dans les deux autres. Cette baisse de la conductivité est due à l'adsorption de certains cations par les argiles. Les charges négatives à la surface des argiles due à la substitution isomorphique, la capacité d'échange cationique élevée des argiles contribuent à la rétention des cations. Ces déchets liquides sont chargés en majorité d'ions $\mathrm{Pb}^{2+}$ issus de l'acétate de plomb basique utilisé pour la clarification des jus de canne à sucre. Les résultats montrent également une forte réduction de la turbidité et de la matière en suspension (MES) suite aux traitements des rejets liquides par les argiles. La turbidité et la MES des déchets liquides sont dues à la présence de fines fibres de canne à sucre dans les jus analysés. Une faible part de la turbidité peut être due à la présence de matières colloïdales d'origine organique ou minérale contenus dans les rejets. Le taux élevé de l'élimination de ces éléments montre qu'ils ont une forte affinité pour les surfaces argileuses. Cette affinité est plus marquée avec l'argile de Katiola que celle d'Anyama. La réduction de la turbidité des eaux usées par les argiles est rapportée dans la littérature (Abdelaal et al., 2010).

L'analyse des teneurs en phosphore total et azote total des rejets liquides, suite au traitement à l'argile, révèle dans l'ensemble une bonne rétention du phosphate et des nitrates présents dans ces rejets. Les argiles utilisées contiennent des minéraux argileux comme l'illite, la Chlorite, et la kaolinite qui sont des minéraux potentiellement favorables à la rétention des phosphates et des nitrates. Par exemple les travaux de Coulibaly et al. (2016) montrent un abattement des phosphates des eaux usées par adsorption des géomatériaux tels que les latérites, le grès et schistes qui contiennent ces mêmes minéraux argileux. Ces minéraux font d'eux des matériaux potentiellement favorables pour la réduction de la pollution phosphatée des eaux.

Les métaux toxiques (cuivre, zinc, mercure et plomb) sont éliminés des déchets liquides à divers degrés et selon l'origine des argiles. Les meilleurs rendements d'éliminations ont été obtenus pour le plomb suivi du cuivre puis du zinc et du mercure. Parmi les quatre métaux lourds le plomb semble s'adsorber préférentiellement sur les argiles devant les trois autres. Le mercure est le métal qui s'adsorbe le mieux parmi les métaux lourds étudiés. Des cas d'adsorption préférentiel ont été rapporté dans la littérature. Il a été rapporté dans une étude (Sdiri et al., 2014) que le cuivre prédominait sur le zinc dans l'adsorption sur une argile naturelle. Une étude d'adsorption montre que le plomb s'adsorbe plus que le cuivre sur des argiles naturelles ou activées (Budsaereechai et al., 2012).

\section{Conclusion}

En somme, les résultats de cette étude ont montré que les argiles d'Anyama et de Katiola, utilisées pour le traitement des déchets liquides sont efficaces pour l'adsorption des polluants organiques et inorganiques. L'argile de Katiola présente de meilleurs rendements comparés à celles d'Anyama et pourrait être recommandée dans le traitement des rejets liquides des laboratoires de l'UAI de Zuénoula. Par ailleurs les résultats obtenus lors de cette étude confirment l'intérêt pratique de l'utilisation des argiles dans le domaine de la dépollution des eaux contaminées par les polluants.

\section{CONFLIT D'INTÉRÊTS}

Les auteurs déclarent qu'il n'y a pas de conflit d'intérêts au sujet de ce travail.

\section{CONTRIBUTIONS DES AUTEURS}

La rédaction a été principalement faite par ABBK. La présentation des tableaux et figures a été faites par SSK. VC et JS ont 
contribué à la conception et la correction de ce document.

\section{REMERCIEMENTS}

Nous remercions l'Unité Agricole Intégrée (UAI) de Zuénoula pour le financement des analyses et le laboratoire de Constitution et de Réaction de la Matière de l'Université Félix Houphouët-Boigny pour les échantillons d'argiles.

\section{RÉFÉRENCES}

Abdelaal A, Gad P, Salem A, Abdelsameia E. 2010. Refineries Wastewaters Treatment by Kaolin Clay. ECWATECH: Moscow, Russia.

Bailey SE, Olin TJ, Bricka RM, Adrian DD. Adrian. 1999. A review of potentially low-cost sorbents for heavy metals. Water Res., 33(11): 2469-2479. DOI: http://dx.doi.org/10.1016/S00431354(98)00475-8

Bhattacharyya KG, Gupta SS. 2008. Adsorption of a few heavy metals on natural and modified kaolinite and montmorillonite: A review. Adv. Colloid Interface Sci., 140(2): 114-131. DOI: http://dx.doi.org/10.1016/j.cis.2007.12.0 08

Budsaereechai S, Kamwialisak K, Ngernyen Y. 2012. Adsorption of lead, cadmium and copper on natural and acid activated bentonite clay. KKU Res., J., 17(5): 800810.

Coulibaly LS, Akpo SK, Yvon J, Coulibaly L. 2016. Fourier transform infra-red (FTIR) spectroscopy investigation, dose effect, kinetics and adsorption capacity of phosphate from aqueous solution onto laterite and sandstone. J. Environ. Manage., 183: 1032-1040. DOI: http://dx.doi.org/10.1016/j.jenvman.2016 .09 .061

Coulibaly V, Kouamé N, Kedi AB, Sei J, Oyetola S. 2018. Reactivity of Clays Consummated in Côte d'Ivoire in Digestive Conditions: Bioavailability of
Mineral Elements. Asian J. Appl. Sci., 6(5): $\quad 321-332 . \quad$ DOI: http://dx.doi.org/10.24203/ajas.v6i5.536 2

Coulibaly V, Sei J, Oyetolas S, Jdid E-A, Thomas F, Yvon J. 2013. Mineralogy and Physicochemical Properties of the Green Clay of Anyama (Côte d'Ivoire) used up for its Healing Properties. Eur. J. Sci. Res., 99(2): 261-270.

Dongo K, Niamke B, Adje A, Britton B, Nama L, Anoh K, Adima A, Atta K. 2013. Impacts des effluents liquides industriels sur l'environnement urbain d'Abidjan Côte D'Ivoire. Int. J. Biol. Chem. Sci., 7(1): $\quad 404 . \quad$ DOI: http://dx.doi.org/10.4314/ijbcs.v7i1.36

Gnagne Y, Yapo B, Meite L, Kouame V, Gadji A, Mambo V, Houenou P. 2015. Caractérisation physico-chimique et bactériologique des eaux usées brutes du réseau d'égout de la ville d'Abidjan. Int. J. Biol. Chem. Sci., 9(2): 1082-1093. DOI:

http://dx.doi.org/10.4314/ijbcs.v9i2.44

Gu S, Kang X, Wang L, Lichtfouse E, Wang C. 2019. Clay mineral adsorbents for heavy metal removal from wastewater: a review. Environ. Chem. Lett., 17(2): 629-654. DOI: http://dx.doi.org/10.1007/s10311018-0813-9

Hu B, Luo H. 2010. Adsorption of hexavalent chromium onto montmorillonite modified with hydroxyaluminum and cetyltrimethylammonium bromide. Appl. Surf. Sci., 257(3): 769-775. DOI: http://dx.doi.org/10.1016/j.apsusc.2010.0 7.062

Kpangni EB, Andji YYJ, Adouby K, Oyetola S, Kra G, Yvon J. 2008. Mineralogy of Clay Raw Materials from Cote d'ivoire: Case of the Deposit from Katiola. J. Appl. Sci., 8(5): 871-875. DOI: http://dx.doi.org/10.3923/jas.2008.871.8 75

MEF (Ministère de l'Environnement et de la Foret). 2008. Arrêté nº1164 du 04 
novembre 2008, portant sur la réglementation des Rejets et Émissions des Installations Classées pour la protection de l'Environnement. Abidjan, Côte d'Ivoire.

Mockovčiaková A, Orolínová Z, Škvarla J. 2010. Enhancement of the bentonite sorption properties. J. Hazard. Mater., 180(1-3): 274-281. DOI: http://dx.doi.org/10.1016/j.jhazmat.2010. 04.027

Ouedraogo DB, Gnankambary Z, Nacro HB, Sedogo MP. 2019. Caractérisation et utilisation des eaux usées en horticulture dans la ville de Ouagadougou au Burkina Faso. Int. J. Biol. Chem. Sci., 12(6): 2564-2577.

DOI: http://dx.doi.org/10.4314/ijbcs.v12i6.8

Ren X, Zhang Z, Luo H, Hu B, Dang Z, Yang C, Li L. 2014. Adsorption of arsenic on modified montmorillonite. Appl. Clay Sci., 97-98: 17-23. DOI: http://dx.doi.org/10.1016/j.clay.2014.05. 028
Sdiri AT, Higashi T, Jamoussi F. 2014. Adsorption of copper and zinc onto natural clay in single and binary systems. Int. J. Environ. Sci. Technol., 11(4): 1081-1092.

DOI: http://dx.doi.org/10.1007/s13762-0130305-1

Sorgho B, Guel B, Zerbo L, Gomina M, Blanchart P. 2019. A study of adsorption of cadmium, copper and lead by two clays from Burkina Faso. Int. J. Biol. Chem. Sci,. 12(6): 2933-2950. DOI: http://dx.doi.org/10.4314/ijbcs.v12i6.35

Yuan GD, Theng BKG, Churchman GJ, Gates WP. 2013. Clays and Clay Minerals for Pollution Control. In Developments in Clay Science, Bergaya F, Lagaly G (eds). Netherlands ; 587-644. DOI : http://dx.doi.org/10.1016/B978-0-08098259-5.00021-4. 